\title{
Vitomir Šunjić and Michael J. Parnham: Signposts to chiral drugs: organic synthesis in action
}

\author{
Springer Basel 2011; ISBN: 978-3-0348-0124-9; e-ISBN: 978-3-0348-0125-6; 232 pages
}

\author{
D. W. Allen
}

Published online: 24 September 2011

(C) Springer Basel AG 2011

This relatively slim volume will be of interest to those working in synthetic organic chemistry, particularly in the medicinal chemistry area, and having as their aim the discovery of new drug entities for the pharmaceutical industries. It is co-written by an academic (VŠ), having considerable experience of teaching and research in synthetic organic chemistry allied to medicinal chemistry, and a pharmacologist (MJP) who has extensive experience of research and development involving a variety of therapeutic agents (including anti-inflammatory drugs) in an industrial context.

In an introductory chapter, the authors present an overview of the challenges facing organic synthesis in the search for new drug entities and point out the importance of chiral synthesis in the design of molecules able to bind to three dimensional biological target sites, recognising that almost $70 \%$ of the top 200 marketed drugs are optically pure. The remainder of the book provides 15 self-contained chapters, each focusing on a specific chiral drug structure, describing its therapeutic profile in terms of its biological target and then presenting a detailed discussion of synthetic approaches to the molecule, the creation of chiral sites and chiral separation techniques. In some cases, retrosynthetic pathways are discussed in relation to potential synthetic strategies and the use of modern reagents, homogeneous catalytic processes and the all-important scaling-up considerations, are highlighted. The range of examples chosen covers a very wide range of therapeutic areas, including anti-inflammatory and anticancer drugs, hypertension and blood lipid control, the treatment of type 2 diabetes, HIV-1, depression and anxiety, antibacterial and anti-asthmatic agents, anti-histamines and even the common household aid (-)-Menthol. Also included is a chapter on 3-amino-1,4benzodiazepines and their potential in the treatment of Alzheimer's disease. At the end of the book, there is also a useful summary of synthetic methods and concepts that are developed in the individual chapters, together with a useful index. The standard of presentation is very high, with many clearly presented reaction schemes, and each chapter has a comprehensive list of references to the specific area.
D. W. Allen $(\bowtie)$

Biomedical Research Centre, Faculty of Health \& Wellbeing, Sheffield Hallam University, Howard Street,

Sheffield S1 1WB, UK

e-mail: d.w.allen@shu.ac.uk 